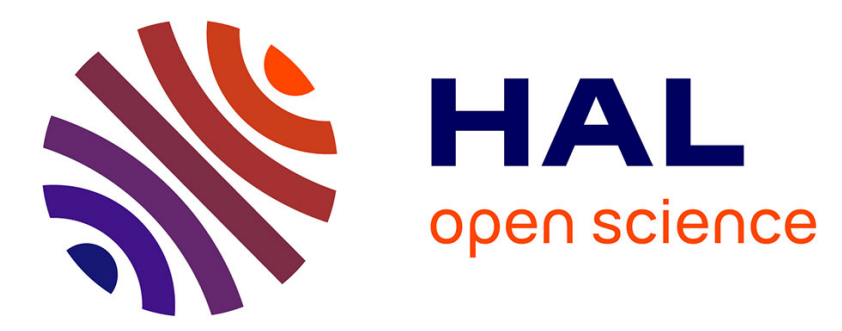

\title{
Mesures de vitesses acoustiques dans les aérogels de silice
}

\author{
P. Hannoun, V. Gibiat, T. Woygnier, J. Pelous
}

\section{To cite this version:}

P. Hannoun, V. Gibiat, T. Woygnier, J. Pelous. Mesures de vitesses acoustiques dans les aérogels de silice. Journal de Physique IV Proceedings, 1994, 04 (C5), pp.C7-229-C7-232. 10.1051/jp4:1994545 . jpa-00253040

\section{HAL Id: jpa-00253040 https://hal.science/jpa-00253040}

Submitted on 1 Jan 1994

HAL is a multi-disciplinary open access archive for the deposit and dissemination of scientific research documents, whether they are published or not. The documents may come from teaching and research institutions in France or abroad, or from public or private research centers.
L'archive ouverte pluridisciplinaire HAL, est destinée au dépôt et à la diffusion de documents scientifiques de niveau recherche, publiés ou non, émanant des établissements d'enseignement et de recherche français ou étrangers, des laboratoires publics ou privés. 


\title{
Mesures de vitesses acoustiques dans les aérogels de silice
}

\author{
P. HANNOUN, V. GIBIAT, T. WOYGNIER* et J. PELOUS ${ }^{*}$ \\ LOA/ESPCI, 10 rue Vauquelin, 75005 Paris, France \\ * LSMV, Montpellier II, Place Eugène Bataillon, 34095 Montpellier, France
}

abstract : The use of silica aerogels as a quarter wave layer for impedance matching of air acoustical transducers has been pointed by FRICKE and AL. in 1986. Their recent published results in 1992 proves the ability and the interest of this approach. Following the same idea, if one want to built efficient transducers, one need precise mesurements of the acoustical properties of a great number of silica aerogel samples. We have choosen a systematical way to verify their properties and find pregnant materials to obtain a good impedance matching in the low ultrasonic range ( $20 \mathrm{kHz}-300 \mathrm{kHz}$ ) between piezoelectric material and air. Mesured acoustical impedance and sound velocity of a wide range of silica aerogel will be presented as preliminar results.

\section{INTRODUCTION}

Les matériaux aérogels ont, depuis leur découverte, suscité de nombreuses tentatives d'utilisations. Parmi celles-ci les applications dans le domaine de l'acoustique sont souvent citées [1,2]. Mais on possède peu d'informations dans ce domaine sur les propriétés de ces matériaux très particuliers. L'étude de leurs propriétés optiques et électromagnétiques, de diffusion des neutrons et des rayons $\mathrm{X}$ sont souvent citées et ont permis de nombreuses avancées dans la compréhension de leur structure, mais peu de travaux ont été publiés dans le domaine sonore et ultrasonore, hormis les travaux du groupe de Zarembowitch [2] et plus récemment ceux du groupe de Fricke [1,3]. Pourtant les applications envisagées sont prometteuses et nécessitent une connaissance approfondie de l'acoustique dans ces matériaux. Dans un double but, celui de la réalisation de lames adaptatrices d'impédance entre une céramique piézo-électrique et le milieu aérien d'une part et celui de la conception d'une terminaison anéchoïque destinée à la calibration d'un dispositif de mesure d'impédance d'autre part, nous avons entrepris la détermination systématique de la vitesse de propagation pour un nombre important d'échantillons. Ce travail a été possible grâce à la collaboration entre nos deux groupes, l'un spécialisé dans l'élaboration de matériaux aérogels et l'autre dans l'acoustique. Nous verrons plus loin que ces mesures nous ont conduit à des interrogations importantes quand aux conditions de propagation et aux possibilités de modélisation de celle-ci.

\section{MATERIAUX AEROGELS ET UTILISATION ACOUSTIQUE.}

Les matériaux aérogels sont obtenus par polymérisation d'un gel de silice en milieu initial neutre ou basique. Après élimination de l'alcool on obtient un aérogel, matériau très léger et dont la porosité peut atteindre voire dépasser $95 \%$. Des études ont montré [4] que la structure des ces porosités était fractale. Les propriétés mécaniques de matériaux aussi poreux sont très mauvaises et on emploie souvent des traitements thermiques pour les améliorer. La structure s'effondre et la fractalité du matériau disparaît très 
vite. Les aérogels peuvent ainsi être densifiés jusqu'à ré obtenir un verre. Les vitesses de propagation des ondes acoustiques peuvent être très faibles. C'est ce qui a été observé jusque alors dans des domaines de très hautes fréquences [2] ou dans des domaines de fréquences plus faibles [1].

Malgré l'existence de ces résultats il reste nombre d'incertitudes sur la dépendance en fréquence de ces mesures qui ont été effectuées pour des bandes fréquentielles limitées. A priori la taille des pores dans un aérogel de silice étant de l'ordre du nanomètre il semble licite d'assimiler le matériau à un solide homogène pour des longueurs d'ondes grandes devant ces dimensions. C'est le cas pour les fréquences ultrasonores inférieures au mégahertz que nous emploierons. Nous considérerons donc dans un premier temps l'aérogel comme un solide homogène de faible densité et pour lequel la célérité des ondes acoustiques est faible. Son impédance acoustique $Z_{a}=\rho c$ est alors très faible. Un calcul simple montre que l'on peut donc concevoir des lames quart d'onde pour adapter l'impédance entre l'air et une céramique.

En effet, si on réduit le problème à la propagation d'une onde plane à travers trois milieux à interfaces parallèles, l'impédance optimale de la lame quart d'onde correspond à l'annulation du coefficient de réflexion à l'interface céramique piézo-électrique - aérogel [5].

Dans le cas général la valeur de ce coefficient $r$ est :

$$
r=\frac{\left(Z_{o} / Z_{p}-1\right)+i\left(Z_{a} / Z_{p}-Z_{o} / Z_{a}\right) \tan \left(k_{a} d\right)}{\left(Z_{o} / Z_{p}+1\right)+i\left(Z_{a} / Z_{p}+Z_{o} / Z_{a}\right) \tan \left(k_{a} d\right)}
$$

On obtient $r=0$ pour un aérogel

d'épaisseur

$$
\mathrm{d}=\lambda / 4
$$

et d'impédance

$$
Z_{a}=\sqrt{Z_{p} \cdot Z_{o}}
$$

La transmission dans l'air reste dans le rapport $Z_{o} / Z_{a}$. C'est-à-dire autour de 100, au lieu de 10.000 pour une interface céramique-air. Ce résultat n'est évidement valable qu'en régime monochromatique. Mais on pourrait aussi examiner la juxtaposition de lames en $\lambda_{i} / 4$ pour une série de fréquence $f_{i}$ dont l'impédance serait $Z_{i}=\sqrt{Z_{i+1} \cdot Z_{i-1}}$; ou mieux une certaine épaisseur d'aérogel possédant un gradient d'impédance. On voit donc l'importance de connaître les vitesses acoustiques des aérogels. Nous avons étudié un nombre très important d'échantillons de densités variées et de modes de préparation aussi variées (basiques , neutres et densifiés).

\section{DISPOSITIF EXPERIMENTAL.}

Le dispositif expérimental utilisé est très classique. Deux transducteurs ultrasonores, de fréquences centrales $140 \mathrm{kHz}$, sont placés de part et d'autre de l'échantillon d'aérogel. La mesure du temps de propagation entre les deux transducteurs conduit pour un matériau homogène à la vitesse connaissant la longueur de l'échantillon. Ce mode de détermination suppose un certain nombre d'hypothèses.

La première est la propagation en mode plan dans le matériau. Nous négligerons ici tous les autres modes, ainsi que les éventuels couplages.

Elle impose également un parallélisme parfait des faces des transducteurs et de l'échantillon.

Le signal imposé à l'entrée du dispositif doit permettre une mesure précise pour l'ensemble des fréquences choisies. Il s'agira donc soit d'une impulsion brève soit d'un signal de type chirp. Nous verrons plus loin les avantages et inconvénients des deux techniques.

Les signaux sont émis par un dispositif programmable qui permet de modifier leur durée et leur forme et mesurés à l'aide d'un oscilloscope numérique sur 12 bits et à une fréquence d'échantillonnage de $2 \mathrm{MHz}$.

\section{TRAITEMENT DES SIGNAUX MESURES.}

La mesure d'un temps de propagation peut se ramener à la détermination d'un déphasage entre le signal d'entrée et le signal de sortie du système.

Soient $s_{1}(t)$ le signal d'émission envoyé au premier transducteur, $s_{2}(t)$ le signal de réception du deuxième transducteur en contact direct avec le premier :

$$
s_{1}(t)=s_{1}(t) * e_{1}(t) * e_{2}(t) \quad \text { (4) où } e_{1}(t) \text { et } e_{2}(t)
$$


sont les réponses impulsionnelles des deux transducteurs. Si on place un échantillon d'aérogel entre les deux, le signal de sortie devient:

$$
s_{2 a}(t)=s_{1}(t) * e_{1}(t) * h_{a}(t) * e_{2}(t) \quad(5) \text { où } h_{a}(t)
$$

est la réponse impulsionnelle de l'échantillon d'aérogel seul, signal qui contient linformation de temps de propagation lié à la longueur $\mathrm{L}$ de l'échantillon. Par transformation de Fourier, le décalage temporel $\tau$ est converti en variation de phase $\omega \tau$

$$
h_{a}(\omega)=s_{2 a}(\omega) / s_{2}(\omega) \text { avec } \arg \left[h_{a}(\omega)\right]=\omega \tau=\theta \quad(6) .
$$

On peut en déduire le décalage temporel en fonction de la fréquence :

$$
\tau(\omega)=\frac{d \theta}{d \omega} \quad(7)
$$

qui est lié à la célérité dans l'aérogel par :

$$
c(\omega)=L / \tau(\omega)
$$

Pour obtenir un résultat sur la célérité en fonction d'une gamme de fréquence la plus large possible, une impulsion en entrée n'est pas le meilleur signal car elle ne fournit pas assez d'énergie, tandis qu'un chirp fournit une énergie plus importante dans sa gamme de fréquence que nous avons limitée à l'intervalle $20 \mathrm{kHz}, 200 \mathrm{kHz}$. Idéalement cela nécessite aussi que les transducteurs aient au moins la même largeur de bande - ce qui n'est pas exactement réalisé pour le moment.

\section{RESULTATS EXPERIMENTAUX.}

Nos résultats sont donnés sous forme de courbes qui représentent l'évolution du déphasage $\theta(\omega)$ (qui varie entre -10 et +10 radians par unité de longueur de l'échantillon) en fonction de la fréquence. Théoriquement la pente de cette courbe est équivalente à l'inverse de la vitesse acoustique dans l'échantillon considéré. Une vitesse indépendante de la fréquence doit donc se traduire par une droite, de pente d'autant plus faible que la vitesse est élevée. A l'inverse, si la vitesse n'est pas constante on doit obtenir des variations dans la pente.

Les résultats obtenus pour trois types d'aérogels : neutre, basique, et densifié semblent typique de ces catégories (figure 1).

Les densités sont pour le densifié de .18 , le neutre .20 et le basique .09 .

Le densifié présente un comportement linéaire presque parfait, qui conduit à une vitesse constante entre 20 $\mathrm{kHz}$ et $200 \mathrm{kHz}$. de $215 \mathrm{~ms}^{-1}$, ce qui correspond bien à la vitesse déterminée par une mesure de propagation d'impulsion. Les quelques accidents de pentes sur la courbe de phase ne semblent pas significatifs en ce qu'ils ne perturbent pas de manière continue cette pente.

Le neutre présente lui aussi un comportement très régulier qui conduit à une vitesse constante légèrement supérieure de $275 \mathrm{~ms}^{-1}$. En revanche le basique se comporte assez différemment en ce sens que la courbe de phase présente de légères variations de pente avec la fréquence : plus faible dans le bas et le haut de la zone étudiée, donc des vitesses plus grandes. La seule partie où on puisse attribuer une pente moyenne qui conduise à un résultat proche de celui obtenu lors d'une mesure de propagation d'impulsion est situé au voisinage de $100 \mathrm{kHz}$. et correspond à une vitesse de $105 \mathrm{~ms}^{-1}$. La vitesse dans les zones à pente faible étant jusqu'à trois fois plus grande.

\section{CONCLUSION.}

Ces variations de pentes se retrouvent sur tous les échantillons basiques que nous avons étudiés ainsi que sur de rares densifiés. Nous n'avons pas d'explication, mais il pourrait s'agir d'erreurs expérimentales liées soit au faible niveau de signal dans ces zones de fréquences, soit au bruit expérimental ou encore à des couplages entre modes... à moins que ce phénomène soit réellement lié à la propagation dans un matériau poreux particulier, car il n'existe pas pour les deux autres groupes.

Les résultats de mesures de vitesses pour l'ensemble des échantillons sont regroupés sur la figure 2 en fonction de la densité. Il est clair que le comportement général est un accroissement de la vitesse avec la fréquence, ce qui exclus une interprétation de ces vitesses lentes par la théorie de Biot [6] des milieux poreux. 

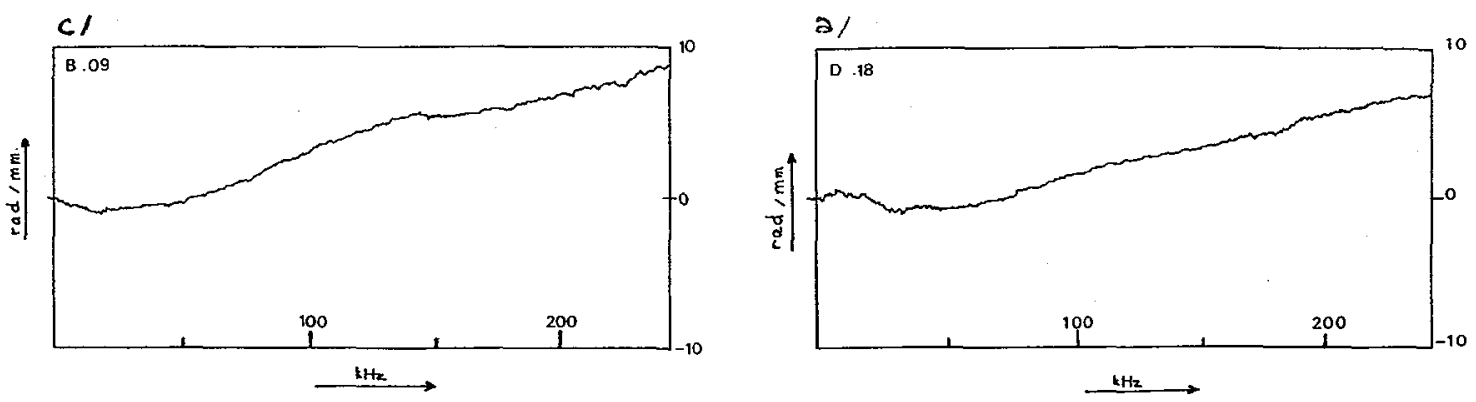

figure 1 : déphasage $Q(f)$ lié à la propagation dans $1 \mathrm{~mm}$. d'aérogel. a) densifié . b) neutre c) basique.

figure 2 : vitesses acoustiques mesurées sur trois types d'aérogels en fonction de la densité.
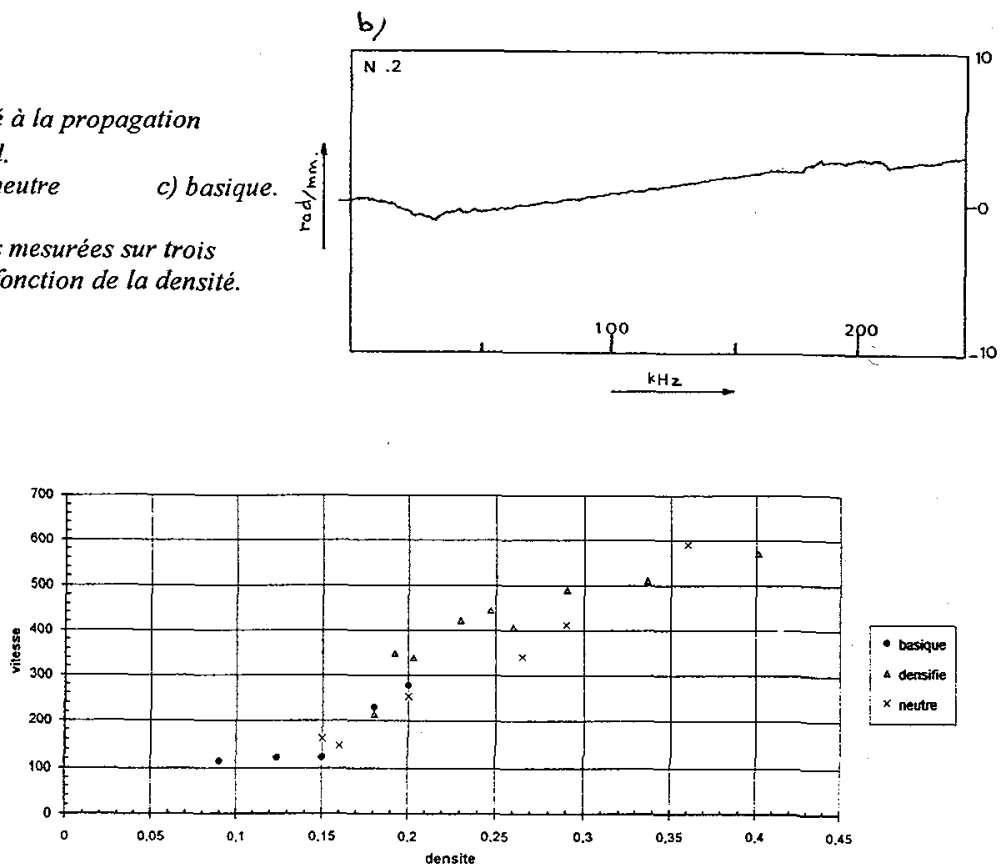

\section{REFERENCES :}

[1] J. Gross and J. Fricke, "Ultrasonic velocity measurements in silica, carbon and organic aerogels". J. Non-Cryst. Solids 145 (1992) p. 217-222

[2] B. Nouailhas, F. Michard, R: Gohier, A.Zarembowitch, "Propagation ultrasonore en milieu très poreux". 1le ICA vol 2. (1983) p.179-182

[3] M.Gronauer and J.Fricke, "Acoustic properties of microporous SiO2-aerogel". Acustica 59 (1986) 177-181

[4] R.Vacher, T.Woignier, J.Pelous, E.Courtens, "Structure and self-similarity of silica aerogels". Phys. REV.B 37 (1988) 6500-3.

[5] R. Gerlach, O. Krauss and J. Fricke, P.-Ch. Esccardt, N. Kroemer and V. Magori, "Modified Sio2 "Aerogels as acoustic impédance matching layers in ullasonic device". J. Non-Crist. Solids 145 (1992) 227-232.

[6] Biot, M.A.,"The theory of propagation of elastic waves in a fluid-saturated porous solid", J. Accoust. Soc. AM., 28, (1956) p.168-191. 